RICYDE. Revista Internacional de Ciencias del Deporte doi: $10.5232 /$ ricyde

Rev. int. cienc. deporte

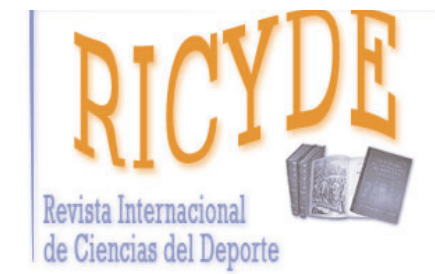

RICYDE. Revista Internacional de Ciencias del Deporte VOLUMEN XI - ANO XI

Páginas:360-375 ISSN:1885-3137

Número 42 - Octubre - 2015

\title{
Efectos del número de jugadores sobre la percepción subjetiva del esfuerzo, la frecuencia cardiaca y las conductas de juego en rugby \\ Number of players' effect on perceived exertion, heart rate and tactical behavior in rugby
}

\author{
Blanca Muñoz-Chavez ${ }^{1}$, Rafael E. Reigal ${ }^{2}$ \\ Antonio Hernández-Mendo ${ }^{1}$, María J. Raimundi ${ }^{3}$
}

1. Facultad de Psicología, Universidad de Málaga. España

2. Grupo Investigación CTS-642 (IDAFISAD), Universidad de Granada. España

3. Consejo Nacional de Investigaciones Científicas y Técnicas (CONICET). Facultad de Psicología, Universidad de Buenos Aires. Argentina

\section{Resumen}

El propósito de este trabajo fue analizar los efectos de diferentes juegos reducidos (JR) sobre la percepción subjetiva del esfuerzo (PSE), la frecuencia cardiaca (FC) y las conductas de juego (CJ) en una muestra de jugadoras de rugby. Participaron 20 jugadoras del equipo de rugby femenino de la Universidad de Málaga (UMA), con edades entre los 16 y 35 años $(M=23.85 ; D T=4.67)$. Para evaluar la PSE se utilizó la Escala de Borg (6 - 20), así como el sistema de monitorización de Firstbeat Sports para analizar la FC y el porcentaje de FC máxima (\% FCmáx) durante los JR. Asimismo, los JR fueron grabados para analizar las CJ. Se desarrollaron tres JR con el mismo tamaño de área por jugador $\left(30 \mathrm{~m}^{2}\right), 3$ vs $3\left(180 \mathrm{~m}^{2}\right), 4$ vs $4\left(240 \mathrm{~m}^{2}\right)$ y 5 vs $5\left(300 \mathrm{~m}^{2}\right)$. Cada formato de juego se distribuyó en cuatro series de cinco minutos de juego, con dos minutos de recuperación entre cada una de ellos. Los resultados indicaron que el juego reducido 3 vs 3 es la modalidad que generó mayores niveles de PSE, FC, \% FCmáx y un mayor número de CJ. La utilización de los JR puede constituir una herramienta eficaz para el trabajo técnico, táctico y físico en el deporte.

Palabras clave: juegos reducidos; percepción subjetiva del esfuerzo; frecuencia cardiaca; acciones de juego; rugby.

\begin{abstract}
The purpose of this study was to analyze the effects of different small-sided games (SSG) on rating perceived exertion (RPE), heart rate (HR) and tactical behavior (TB) in a sample of rugby players. Twenty players from the female team of the University of Malaga (UMA) participated, aged between 16 and 35 years $(M=23.85, S D=4.67)$. The Borg Scale $(6-20)$ was used to assess RPE, Firstbeat Sports monitoring system was used to analyze HR and maximum heart rate percentage (\% HRmax) during SSG. Moreover, SSG were videotaped to analyze TB. Three SSG with the same size of area per player $\left(30 \mathrm{~m}^{2}\right), 3$ vs. $3\left(180 \mathrm{~m}^{2}\right), 4$ vs. $4\left(240 \mathrm{~m}^{2}\right)$ and 5 vs $5\left(300 \mathrm{~m}^{2}\right)$ were developed. Each game format was distributed in four series of five minutes, with two rest minutes between each of them. Results showed that the SSG 3 vs 3 is the modality that generated higher levels of RPE, HR, \% HRmax and a larger number of TB. The use of SSG could be an effective tool for technical, tactical and physical work in sport.
\end{abstract}

Key words: small-sided games; rating perceived exertion; heart rate; tactical behavior; rugby.

Correspondencia/correspondence: Rafael Enrique Reigal Garrido

Grupo Investigación CTS-642 (IDAFISAD), Universidad de Granada. España

Email: rafareigal@gmail.com 
Muñoz-Chaves, B.M.; Reigal, R.E.; Hernández-Mendo, A., y Raimundi, M.J. (2015). Efectos del número de jugadores sobre la percepción subjetiva del esfuerzo, la frecuencia cardiaca y las conductas de juego en rugby. RICYDE. Revista internacional de ciencias del deporte, 42(11), 360-375.

http://dx.doi.org/10.5232/ricyde2015.04205

\section{Introducción}

$\mathrm{L}$ os juegos reducidos (JR) son ejercicios que se están utilizando ampliamente en los entrenamientos de los deportes colectivos (Casamichana, Castellano, y Hernández-Mendo, 2014; Dellal y col., 2012; Sampaio, Lago, Gonçalves, Maçãs, y Leite, 2014). Estas situaciones mantienen la lógica interna del juego pero en ellos se pueden modificar diversos elementos para condicionar la tarea a realizar y alcanzar objetivos determinados (Casamichana y Castellano, 2010; Hill-Haas, Dawson, Impellizzeri, y Coutts, 2011). Por otro lado, los JR son adecuados para desarrollar y transferir conocimientos técnico-tácticos (Buchheit y col., 2009; Gabbett, Jenkins, y Abernethy, 2009). Se ha observado que son efectivos para mejorar aspectos físicos, y al trabajar sobre situaciones de juego con balón, también son útiles para mantener niveles de motivación altos en el deportista (Casamichana, Castellano, González-Morán, García-Cueto, y García-López, 2011; Hoff y Helgerud, 2004).

Algunas de las modificaciones de los JR que han sido más estudiadas son las efectuadas sobre el número de jugadores (Abrantes, Nunes, Maças, Leite, y Sampaio, 2012; Castagna, Impellizzeri, Chaovachi, Abdelkrim, y Manzi, 2011), el tamaño del terreno de juego (Frencken, Van Der Plaats, Visscher, y Lemmink, 2013; Köklü, Albayrak, Keysan, Alemdaroglu, y Dellal, 2013), la duración del ejercicio o el tiempo de descanso entre tareas (Dellal, Drust, y Lago-Penas, 2012; Duarte, Batalha, Folgado, y Sampaio, 2009; Fanchini y col., 2011), las reglas de juego (HillHaas, Coutts, Dawson, y Rowsell, 2010; Vickery, Dascombe, Duffield, Kellet, y Portus, 2013), la inclusión del portero o la inclusión de porterías en aquellos deportes que poseen este elemento (Casamichana, Castellano, González-Morán, García-Cueto, y García-López, 2011; Dellal y col., 2008, 2012) o la presencia activa del entrenador (Rampinini y col., 2006; Sampaio y col., 2007).

Algunas de las variables más analizadas como consecuencia de estas alteraciones son la frecuencia cardiaca (FC), la percepción subjetiva del esfuerzo (PSE) o las conductas de juego (CJ) (Casamichana y Castellano, 2010; Hill-Haas y col., 2010). Específicamente, se han observado en diferentes investigaciones la existencia de alteraciones significativas en estas variables cuando se modifica el número de jugadores, encontrándose, en general, un efecto consistente en FC y PSE. Aunque existen excepciones, suelen encontrarse niveles más elevados de estos parámetros cuando se reduce el número de jugadores. En el fútbol, por ejemplo, autores como Owen, Wong, McKenna, y Dellal (2011) analizaron en 15 jugadores profesionales que durante el JR 3 vs 3 (JR3) se trabajaba a un porcentaje de frecuencia cardiaca máxima (\% FCmáx) mayor que en el formato 9 vs 9 (JR9) y se producía un mayor número de contactos con el balón. En otro trabajo de Abrantes y col. (2012) encontraron en una muestra de jóvenes jugadores que el JR3 estaba relacionado con mayores niveles de \% FCmáx y PSE que el JR 4 vs 4 (JR4).

En otros deportes, Castagna y col. (2011) encontraron en un grupo de jugadores de baloncesto jóvenes que las modalidades de juego 2 vs 2 (JR2) desarrollaban tasas más elevadas de \% FCmáx que JR3, y éste a su vez mayores que JR4. Por su parte, Sampaio, Abrantes, y Leite (2009) encontraron, en una muestra de jóvenes jugadores de baloncesto, que el JR3 provocaba un trabajo a un mayor \% FCmáx que el JR4, aunque no se apreciaban diferencias significativas en PSE. En un trabajo realizado por Clemente, Rocha, Lourenço, y Sousa (2014) observaron, en una clase de Educación Física y en una muestra de chicas adolescentes que un JR2 de balonmano generaba mayores tasas de FC que un JR3 y JR4. Asimismo, Morales, y Arias-Estero (2015) hallaron en un grupo de escolares adolescentes que un JR4 provocaba mayores niveles de PSE que un JR 7 s 7.

Respecto a las CJ se ha observado, por regla general y en diferentes deportes, un mayor número 
Muñoz-Chaves, B.M.; Reigal, R.E.; Hernández-Mendo, A., y Raimundi, M.J. (2015). Efectos del número de jugadores sobre la percepción subjetiva del esfuerzo, la frecuencia cardiaca y las conductas de juego en rugby. RICYDE. Revista internacional de ciencias del deporte, 42(11), 360-375.

http://dx.doi.org/10.5232/ricyde2015.04205

de intervenciones por jugador en los JR con menor número de jugadores (Kelly y Drust, 2009; Klusemann, Pyne, Foster, y Drinkwater, 2012; Owen y col., 2011). No obstante, para tener una perspectiva más aproximada de cada tipo de acción técnico-táctica hay que efectuar un análisis más específico. De hecho, Owen, Wrong, Paul, y Dellal (2014) hallaron en jugadores de fútbol profesional que el JR4 tuvo mayor frecuencia de pases que las modalidades desde 5 vs 5 (JR5) hasta el formato 11 vs 11 (JR11), y aunque también ocurrió con otras acciones como las recepciones y los lanzamientos, las diferencias no fueron significativas respecto al JR5 y el JR 6 vs 6 (JR6). Asimismo, Da Silva y col. (2011) observaron, en una muestra de jugadores adolescentes de fútbol, que el formato JR3 generaba un mayor número de dribles o lanzamientos que en el JR4 y el JR5, aunque no hubo diferencias significativas en las conductas de pase. Por otro lado, Katis y Kellis (2009) observaron en un grupo de futbolistas adolescentes que el formato JR3 generaba mayor número de pases cortos, lanzamientos, tackles, dribles y goles que en el JR6, aunque menos pases largos y golpeos de cabeza.

En rugby, existen diferentes líneas de investigación que examinan parámetros fisiológicos y conductuales en competición (e.g., Villarejo, Palao, y Ortega, 2011; Villarejo, Gomez, Palao, y Ortega, 2012; Villarejo, Palao, Ortega, Gomez, y Kraak, 2015). Además, algunos investigadores han evaluado los efectos en el entrenamiento que tienen los JR en este deporte, mostrando su efectividad para la mejora del rendimiento en competición (Gabbett, 2006; Seitz, Rivière, de Villarreal, y Haff, 2014). Asimismo, autores como Gabbett, Abernethy, y Jenkins (2012) analizaron en jugadores de élite jóvenes y seniors los efectos de las dimensiones del terreno de juego en diversas variables fisiológicas y acciones de juego. Observaron, para ambas categorías, que los JR en terrenos más amplios generaban una mayor intensidad de trabajo pero no había diferencias en el volumen y calidad de las acciones técnico-tácticas evaluadas. Por otro lado, Foster, Twist, Lamb, y Nicholas (2010) evaluaron dos JR con diferente número de jugadores, señalando que durante el JR4 se obtuvieron mayores \% FCmáx que durante un JR6 en un grupo de adolescentes de 15 y 16 años, aunque no hallaron diferencias significativas en un grupo de 12 y 13 años.

Sin embargo, no existe un cuerpo de conocimiento tan amplio como en otros deportes sobre el uso de los JR en el rugby. Por ello, con el propósito de incrementar la información disponible en este deporte, el objetivo del presente trabajo fue analizar el efecto del número de jugadores (JR3, JR4 y JR5) sobre la FC, el \% FCmáx y la PSE en una muestra de jugadoras de rugby.

\section{Método}

\section{Diseño}

Se trata de un estudio empírico, descriptivo y comparativo (Ato, López y Benavente, 2013), en el que se han analizado el impacto de tres tipos de JR sobre diversos parámetros físiológicos, psicofísicos y conductuales.

\section{Muestra}

Participaron en este estudio 20 jugadoras del equipo de rugby femenino de la Universidad de Málaga (UMA), con edades comprendidas entre los 16 y 35 años $(M \pm D T$ : edad $=23.85 \pm 4.67$ años; altura $=162.90 \pm 6.08 \mathrm{~cm}$; peso $=62.88 \pm 5.83 \mathrm{~kg}$; IMC $=23.61 \pm 2.35 \mathrm{~kg} \cdot \mathrm{m}^{-2}$; masa grasa $=$ $21.76 \pm 3.65 \%$; FCmáx. $=195.80 \pm 7.05 \mathrm{ppm}$; VO2max $=43.09 \pm 3.18 \mathrm{ml} \cdot \mathrm{kg}^{-1} \cdot \mathrm{min}^{-1}$ ). Las jugadoras participaron de forma voluntaria y no presentaban problemas físicos o psicológicos reseñables. Se obtuvo el consentimiento informado de cada jugadora para poder participar, solicitando autorización a los padres/tutores legales en caso de las participantes menores de 
Muñoz-Chaves, B.M.; Reigal, R.E.; Hernández-Mendo, A., y Raimundi, M.J. (2015). Efectos del número de jugadores sobre la percepción subjetiva del esfuerzo, la frecuencia cardiaca y las conductas de juego en rugby. RICYDE. Revista internacional de ciencias del deporte, 42(11), 360-375.

http://dx.doi.org/10.5232/ricyde2015.04205

edad. Las participantes completaron todos los ejercicios, por lo que el tamaño de la muestra para cada uno de los registros y formatos fue $n=20$. Además, durante todo el proceso de investigación se respetaron los principios establecidos en la declaración de Helsinki (2000). La categoría en la que participaba el equipo era de nivel regional en la modalidad rugby-15 y nivel nacional en rugby-7.

\section{Variables}

a) Frecuencia cardiaca (FC). Se obtuvieron valores de FC y \% FCmáx obtenidos durante los JR. Para ello, se utilizó el sistema de monitorización de Firstbeat ${ }^{\circledR}$ Sports Professional System, que registra frecuencia cardiaca en tiempo real y permite monitorizar hasta 10 registros simultáneamente. Se consideró como frecuencia cardiaca máxima el valor más alto alcanzado durante el test de Course Navette (Léger, Mercier, Gadoury y Lambert, 1988), prueba que consiste en un test incremental de ida y vuelta, sobre una distancia de 20 metros, con un aumento de la velocidad de $0.5 \mathrm{~km}$ cada minuto, partiendo de una velocidad inicial de $8.5 \mathrm{~km} / \mathrm{h}$.

b) Conductas de juego. Se analizaron las siguientes acciones: número de intentos de pase (P), número de pases acertados (PA; cuando el pase se completa con la recepción de un compañero), número de pases erróneos (PE; pase adelantado o interceptado por un jugador del equipo contrario), número de placajes (PLA; placajes completos o intentos de placajes que haga detenerse a un oponente), número de interceptaciones (INT; pase cortado por un oponente), número de contra pies (PIE; el jugador cambia la dirección de carrera), número de rucks (RUC; regla 16 de la World Rugby), número de patadas (PAT; jugar la pelota con el pie), número de maul (MAU; regla 17 de la World Rugby), número de ensayos (TRY; llevar y posar la pelota en el extremo contrario del terreno de juego) y número de continuidades (CON; el jugador pasa el balón antes de caer por el placaje).

c) Percepción subjetiva del esfuerzo (PSE). Para evaluar la PSE tras cada periodo de trabajo se utilizó la Escala de Borg 6-20 (Borg, 1973). Esta medida de tipo psicofísico se considera que representa la intensidad del trabajo realizado y se construye mediante un proceso en el que diversos factores influyen en la percepción del esfuerzo llevado a cabo.

\section{Procedimiento}

Para realizar este trabajo se contactó con los responsables del equipo de rugby femenino de la UMA y se solicitó permiso para efectuar la investigación. Las evaluaciones se efectuaron entre febrero y marzo del año 2014, en un intervalo horario similar (entre las 19:00 y las 20:00 horas) y en la misma superficie de juego (hierba artificial).

Las condiciones específicas de cada juego reducido fueron partidos de 3 vs 3 en unas dimensiones de $15 \times 12$ metros (180 m²; $30 \mathrm{~m}^{2}$ /jugador), 4 vs 4 en dimensiones de $17.5 \times 14$ metros $\left(240 \mathrm{~m}^{2} ; 30 \mathrm{~m}^{2} /\right.$ jugador $)$ y 5 vs 5 en dimensiones de 18.75 x 16 metros $\left(300 \mathrm{~m}^{2} ; 30\right.$ $\mathrm{m}^{2} /$ jugador). Cada juego se desarrolló en cuatro periodos de cinco minutos, con dos minutos de recuperación entre cada una de ellos. Los JR estuvieron precedidos por un calentamiento de 10 minutos, que consistió en carrera continua, movilidad articular y ejercicios específicos de juego. Cada modalidad de JR se desarrolló en una sesión independiente con una diferencia de tiempo de una semana.

Inicialmente, cada jugadora realizó el test de esfuerzo incremental Course Navette para calcular su frecuencia cardiaca máxima. Se consideró el valor máximo de $\mathrm{FC}$ alcanzada durante la prueba como la medida de FCmáx, la cual sirvió para considerar el \% FCmáx durante los JR. Durante las diferentes evaluaciones, se monitorizó a cada jugadora para obtener el valor medio de FC en cada periodo, así como el \% FCmáx al que habían trabajado en cada uno de ellos. La 
Muñoz-Chaves, B.M.; Reigal, R.E.; Hernández-Mendo, A., y Raimundi, M.J. (2015). Efectos del número de jugadores sobre la percepción subjetiva del esfuerzo, la frecuencia cardiaca y las conductas de juego en rugby. RICYDE. Revista internacional de ciencias del deporte, 42(11), 360-375.

http://dx.doi.org/10.5232/ricyde2015.04205

monitorización fue simultánea a través de un receptor situado aproximadamente a 25 metros de distancia, el cual enviaba a un ordenador el registro de FC emitido por cada jugadora. La escala de PSE fue rellenada por cada jugadora inmediatamente después de cada periodo de trabajo y almacenada en carpetas individuales para evitar interferencias entre ellas. Para que el dato obtenido fuera fiable, las participantes fueron entrenadas en su uso durante varias semanas previas a las evaluaciones, complementando el aprendizaje a través de lecturas de las instrucciones de la prueba (Feriche, Chicharro, Vaquero, Perez, y Lucia, 1998). Además, se calculó el índice de correlación (Pearson) entre los valores de los diferentes periodos de trabajo, situándose entre .60 y .94 .

Para analizar las CJ todos los partidos fueron grabados en vídeo, con una cámara Canon ${ }^{\circledR}$ LEGRIA FS306. Se situó en una grada anexa al terreno de juego, a una altura de 4 metros de alto y con soporte fijo, que permitía recoger imágenes de todo el desarrollo del juego. Los propios investigadores visionaron los vídeos y registraron las CJ que se habían producido. Para analizar la calidad del dato, se efectuó un análisis de generalizabilidad (Blanco-Villaseñor, Castellano, Hernández-Mendo, Sánchez-López, y Usabiaga, 2014) con un modelo cruzado de tres facetas [j] [p] /[a], donde [j] (tipo de juego) es una faceta de diferenciación con tres niveles y un tamaño muestral infinito, [p] (participante) es una faceta de diferenciación con 20 niveles y un tamaño muestral infinito y [a] (acción de juego) es una faceta de instrumentación con 11 niveles y un tamaño muestral infinito. El índice $\mathrm{G}$ relativo fue .60 , valor que puede ser considerado como aceptable. En relación con las reglas de juego, se aplicaron las recogidas en la World Rugby para el juego abierto. No obstante, para el JR3 se adaptó la regla 16, entendiendo por ruck: un jugador placado y un defensa sobre sus pies que pone el balón en juego.

\section{Análisis de los datos}

Se efectuaron análisis descriptivos e inferenciales para analizar los datos. Se comprobó la normalidad de los mismos a través de la prueba Shapiro-Wilk. Para evaluar la diferencias en entre los grupos se efectuaron análisis de la varianza (ANOVA) para las medidas con distribución normal, utilizando la prueba de Bonferroni para las comparaciones simples. Asimismo, para las distribuciones con problemas de normalidad se analizaron con la prueba de Friedman y el test Wilcoxon. En estos casos, el nivel de significación para las comparaciones simples fueron ajustadas mediante el procedimiento de Bonferroni ( $\alpha / \mathrm{n}^{\mathrm{o}}$ comparaciones), en este caso el $p<.05 / 3(=.016)$. Además, se analizó la fiabilidad de la medida de PSE a través de un análisis de correlaciones mediante el coeficiente bivariado de Pearson. Para el procesamiento estadístico de los datos se ha usado el programa informatizado SPSS en su versión 20.0.

\section{Resultados}

En la tabla 1 se muestran las medias y desviaciones típicas de percepción subjetiva del esfuerzo (PSE), frecuencia cardiaca (FC), porcentaje de frecuencia cardiaca máxima (\% FCmáx) y conductas de juego (CJ). 
Muñoz-Chaves, B.M.; Reigal, R.E.; Hernández-Mendo, A., y Raimundi, M.J. (2015). Efectos del número de jugadores sobre la percepción subjetiva del esfuerzo, la frecuencia cardiaca y las conductas de juego en rugby. RICYDE. Revista internacional de ciencias del deporte, 42(11), 360-375.

http://dx.doi.org/10.5232/ricyde2015.04205

Tabla 1. Medias y desviaciones típicas de percepción subjetiva del esfuerzo, frecuencia cardiaca, porcentaje de frecuencia cardiaca máxima y conductas de juego

\begin{tabular}{|c|c|c|c|c|c|c|c|}
\hline & & \multicolumn{2}{|c|}{$3 v s 3$} & \multicolumn{2}{|c|}{$4 v s 4$} & \multicolumn{2}{|c|}{$5 v s 5$} \\
\hline & & $M$ & $D T$ & $M$ & $D T$ & $M$ & $D T$ \\
\hline \multirow[t]{5}{*}{ PSE } & $\mathrm{P} 1$ & 11.55 & 2.16 & 10.00 & 1.45 & 10.25 & 1.74 \\
\hline & P2 & 12.65 & 2.78 & 10.95 & 1.76 & 11.45 & 1.61 \\
\hline & P3 & 12.95 & 2.09 & 11.70 & 1.78 & 11.95 & 1.23 \\
\hline & P4 & 12.96 & 1.96 & 11.71 & 1.95 & 12.35 & 1.27 \\
\hline & Total & 12.53 & 2.11 & 11.09 & 1.40 & 11.50 & 1.25 \\
\hline \multirow[t]{5}{*}{$\mathrm{FC}$} & P1 & 166.50 & 11.22 & 164.95 & 9.62 & 160.40 & 9.95 \\
\hline & $\mathrm{P} 2$ & 172.75 & 9.08 & 167.45 & 9.16 & 164.60 & 10.89 \\
\hline & P3 & 170.65 & 11.53 & 164.65 & 13.91 & 157.65 & 13.81 \\
\hline & P4 & 168.65 & 10.18 & 165.95 & 11.97 & 154.90 & 16.81 \\
\hline & Total & 169.64 & 9.89 & 165.75 & 9.88 & 159.39 & 10.26 \\
\hline \multirow[t]{5}{*}{$\% \mathrm{FCM}$} & P1 & 84.70 & 6.24 & 83.90 & 4.66 & 81.55 & 5.08 \\
\hline & $\mathrm{P} 2$ & 87.95 & 5.40 & 85.10 & 4.96 & 83.60 & 5.46 \\
\hline & P3 & 86.85 & 6.81 & 83.65 & 7.77 & 80.05 & 7.11 \\
\hline & P4 & 85.70 & 5.75 & 84.35 & 6.45 & 78.75 & 8.68 \\
\hline & Total & 86.30 & 5.77 & 84.25 & 5.39 & 80.99 & 5.28 \\
\hline \multirow[t]{12}{*}{ CJ } & $\mathrm{P}$ & 4.96 & 1.16 & 3.16 & 1.10 & 3.38 & 1.20 \\
\hline & PA & 4.49 & 1.27 & 2.68 & 1.08 & 3.03 & 1.18 \\
\hline & PE & .49 & .33 & .51 & .33 & .35 & .19 \\
\hline & PLA & 2.73 & 1.20 & 2.24 & .88 & 2.61 & 1.20 \\
\hline & INT & .11 & .21 & .06 & .11 & .16 & .25 \\
\hline & PIE & 1.56 & .76 & 1.51 & 1.01 & 1.54 & .98 \\
\hline & RUC & .91 & .76 & .88 & .63 & 1.10 & .65 \\
\hline & PAT & .01 & .06 & .04 & .09 & .03 & .08 \\
\hline & MAU & .08 & .14 & .18 & .16 & .20 & .30 \\
\hline & TRY & 1.75 & .73 & .91 & .60 & .58 & .51 \\
\hline & $\mathrm{CON}$ & .70 & .52 & .59 & .52 & .90 & .37 \\
\hline & Total & 1.62 & .27 & 1.16 & .31 & 1.26 & .30 \\
\hline
\end{tabular}

Nota: PSE = Percepción subjetiva del esfuerzo; FC = Frecuencia cardiaca; \% FCmáx = Porcentaje de frecuencia cardiaca máxima; $\mathrm{P}=$ Periodo; $\mathrm{CJ}=$ conductas de juego; $\mathrm{P}=$ Pases; $\mathrm{PA}=$ Pases acertados; $\mathrm{PE}=$ Pases erróneos; PLA = Placajes; INT = Interceptaciones; PIE = Contra pies; RUC $=$ Rucks; PAT $=$ Patadas $; \mathrm{MAU}=$ Maul $; \mathrm{TRY}=$ Ensayos $; \mathrm{CON}=$ Continuidad.

Los análisis de normalidad indicaron que para la PSE y la FC, los valores de asimetría (entre .69 y .61), curtosis (entre -1.28 y 1.20) y de la prueba Shapiro-Wilk (entre .91 y .98) fueron adecuados. Sin embargo, para el \% FCmáx y las CJ algunos valores mostraron problemas de normalidad (asimetría $=$ entre -1.49 y 4.47 ; curtosis $=$ entre -1.06 y 20 ; Shapiro-Wilk $=$ entre .24 y .98).

En la figura 1 se presentan las comparaciones de grupo realizadas en PSE en función a los diferentes JR. Se han hallado diferencias significativas en PSE entre los diferentes juegos reducidos $(\mathrm{JR})$ en todos los periodos $(\mathrm{P})$ de trabajo $\mathrm{P} 1\left(\mathrm{~F}_{[2,18]}=5.85, p<.05\right), \mathrm{P} 2\left(\mathrm{~F}_{[2,18]}=3.80\right.$, $p<.05), \mathrm{P} 3\left(\mathrm{~F}_{[2,18]}=3.18, p=.06\right), \mathrm{P} 4\left(\mathrm{~F}_{[2,18]}=3.49, p<.05\right)$ y en el valor medio de cada uno de los JR $\left(\mathrm{F}_{[2,18]}=4.93, p<.05\right)$. Las comparaciones simples indicaron un mayor nivel de PSE en el 3 vs 3 respecto al 4 vs 4, aunque las diferencias significativas o próximas a la significación entre 3 vs 3 y 5 vs 5 únicamente se apreciaron en el P1 y en el valor medio de los periodos. 
Muñoz-Chaves, B.M.; Reigal, R.E.; Hernández-Mendo, A., y Raimundi, M.J. (2015). Efectos del número de jugadores sobre la percepción subjetiva del esfuerzo, la frecuencia cardiaca y las conductas de juego en rugby. RICYDE. Revista internacional de ciencias del deporte, 42(11), 360-375.

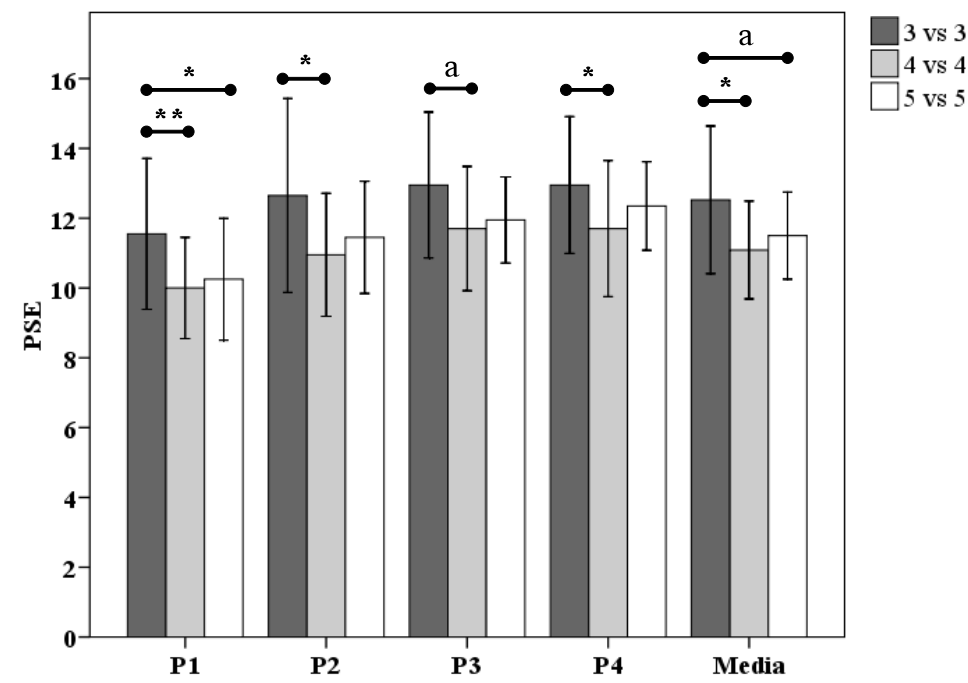

Figura 1. Diferencias en percepción subjetiva del esfuerzo entre los diferentes juegos reducidos (JR) en cada periodo y valor medio Nota: PSE $=$ percepción subjetiva del esfuerzo ${ }^{*} p<.05 ; * *<<.01 ;{ }^{\mathrm{a}} p=.06$

En la figura 2 se presentan las comparaciones de grupo realizadas en FC en función a los diferentes JR. Se hallaron diferencias significativas en FC entre los diferentes juegos reducidos $(\mathrm{JR})$ en los periodos $\mathrm{P} 2\left(\mathrm{~F}_{[2,18]}=11.02, p<.001\right), \mathrm{P} 3\left(\mathrm{~F}_{[2,18]}=6.71, p<.01\right), \mathrm{P} 4\left(\mathrm{~F}_{[2,18]}=6.31, p\right.$ $<.01) \mathrm{y}$ en el valor medio de cada uno de $\operatorname{los} \mathrm{JR}\left(\mathrm{F}_{[2,18]}=9.92, p<.01\right)$. Las comparaciones simples indicaron un mayor valor de FC en el 3 vs 3 respecto al 5 vs 5 en P2, P3, P4 y en el valor medio de los periodos, así como un mayor nivel de FC en el 3 vs 3 respecto al 4 vs 4 en el P2.

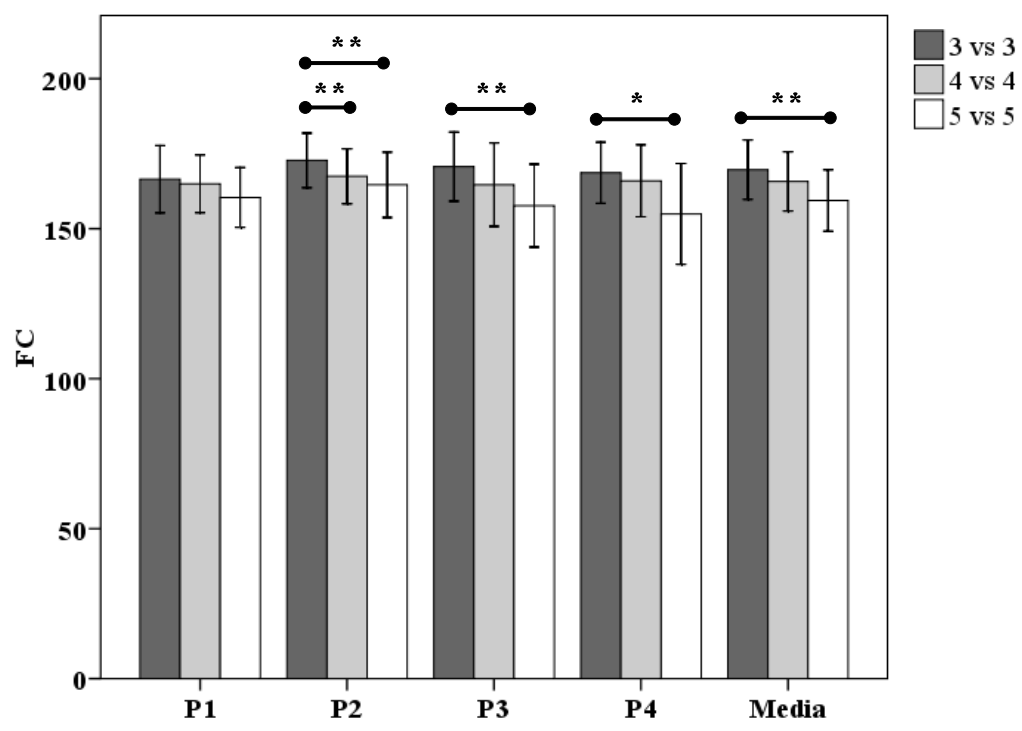

Figura 2. Diferencias en frecuencia cardiaca entre los diferentes juegos reducidos en cada periodo y valor medio

Nota $:$ FC $=$ frecuencia cardiaca ${ }^{*} p<.05 ; * * p<.01 ;{ }^{\mathrm{a}} p=.06$

En la figura 3 se presentan las comparaciones de grupo realizadas en \% FCmáx en función a los 
Muñoz-Chaves, B.M.; Reigal, R.E.; Hernández-Mendo, A., y Raimundi, M.J. (2015). Efectos del número de jugadores sobre la percepción subjetiva del esfuerzo, la frecuencia cardiaca y las conductas de juego en rugby. RICYDE. Revista internacional de ciencias del deporte, 42(11), 360-375.

diferentes JR. Se hallaron diferencias significativas en \% FCmáx entre los diferentes JR en los periodos P2 $\left(x^{2}=9.33, p<.01\right), \mathrm{P} 3\left(x^{2}=7.58, p<.05\right), \mathrm{P} 4\left(x^{2}=5.31, p<.05\right)$ y en el valor medio de cada uno de los JR $\left(x^{2}=10.66, p<.01\right)$. Las comparaciones simples indicaron un mayor valor de \% FCmáx en el 3 vs 3 respecto al 5 vs 5 en P2, P3, P4 y en el valor medio de los periodos, así como entre 3 vs 3 respecto al 4 vs 4 en P2 y en el valor medio.

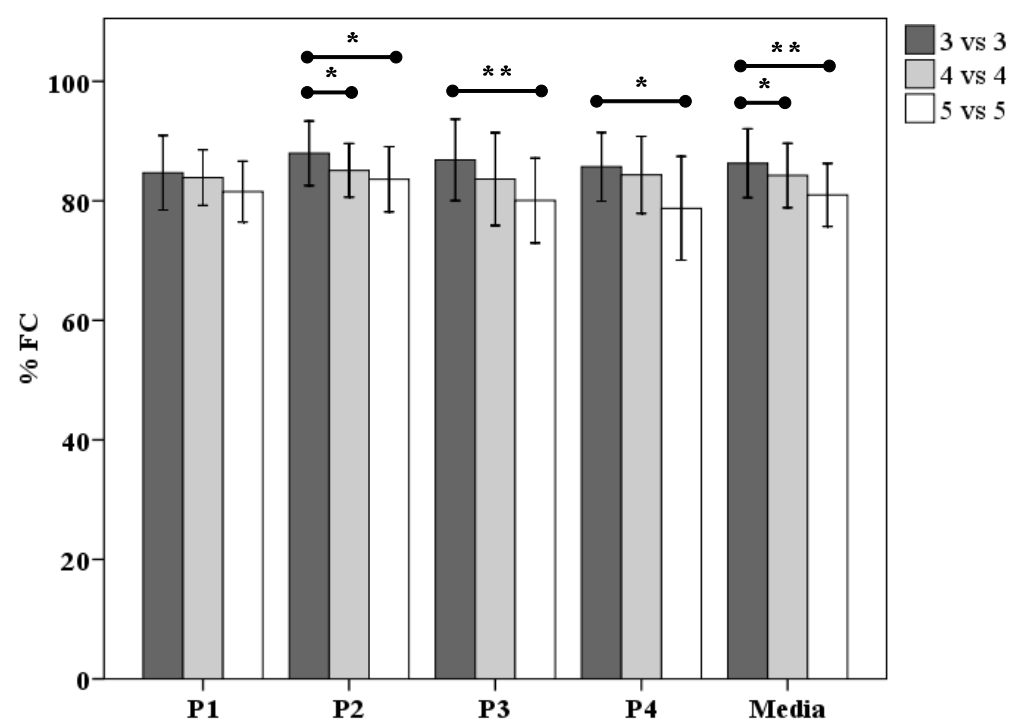

Figura 3. Diferencias porcentaje de frecuencia cardiaca máxima entre los diferentes juegos reducidos en cada periodo y valor medio Nota: $\%$ FCmáx $=$ porcentaje de frecuencia cardiaca máxima ${ }^{*} p<.016 ; * * p<003$ (Nivel de significación ajustado por el procedimiento de Bonferroni)

En la figura 4 se presentan las comparaciones de grupo para las CJ en función a los diferentes JR. Se hallaron diferencias significativas entre los diferentes JR. Las diferencias se dieron en $\mathrm{P}$ $\left(X^{2}=22.94, p<.001\right)$, PA $\left(X^{2}=23.17, p<.001\right)$, TRY $\left(X^{2}=20.41, p<.001\right)$ y para los valores medias de cada JR $\left(X^{2}=26.56, p<.001\right)$, así como indicios de significación en CON $\left(X^{2}=5.51\right.$, $p=.064)$. Las comparaciones simples indicaron un mayor número de pases, pases acertados, ensayos y número medio total de acciones en el $3 v s 3$ respecto al $4 v s 4$ y al $5 v s 5$. Asimismo, hubo indicios de significación en el número de acciones de continuidad entre el 4 vs 4 y el 5 vs 5 a favor del 5 vs 5 . 
Muñoz-Chaves, B.M.; Reigal, R.E.; Hernández-Mendo, A., y Raimundi, M.J. (2015). Efectos del número de jugadores sobre la percepción subjetiva del esfuerzo, la frecuencia cardiaca y las conductas de juego en rugby. RICYDE. Revista internacional de ciencias del deporte, 42(11), 360-375.

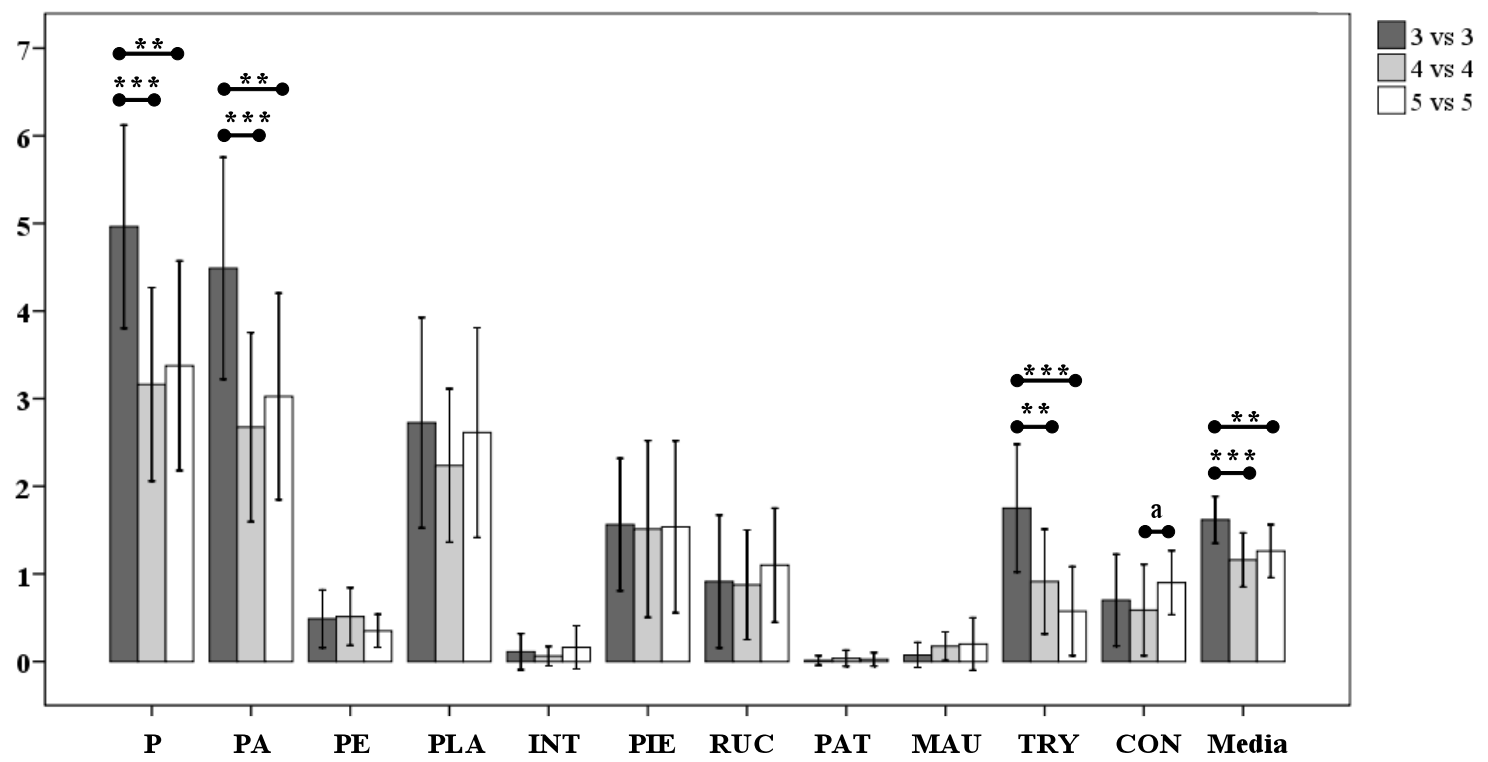

Figura 4. Diferencias número de acciones de juego entre los diferentes juegos reducidos $\mathrm{P}=$ Pases; PA = Pases acertados; PE = Pases erróneos; PLA = Placajes; INT = Interceptaciones; PIE = Contra pies; RUC $=$ Rucks; PAT $=$ Patadas; $\mathrm{MAU}=$ Maul; TRY = Ensayos; $\mathrm{CON}=$ Continuidad $* p<.05 / 3 ; * * p<.01 / 3 ; * * * p<.001 / 3 ;{ }^{a} p=.017$ (Nivel de significación ajustado por el procedimiento de Bonferroni)

\section{Discusión}

El objetivo de este estudio fue evaluar el efecto del número de jugadores sobre la frecuencia cardiaca (FC), la percepción subjetiva del esfuerzo (PSE) y las conductas de juego (CJ) en una muestra de jugadoras de rugby. Para ello se analizaron tres juegos reducidos (JR) con formatos 3 $v s 3$ (JR3), 4 vs 4 (JR4) y 5 vs 5 (JR5), en un área de juego de $30 \mathrm{~m}^{2}$ por jugador en todos los casos. Los resultados obtenidos pusieron de relieve que la modificación del número de jugadores generaba diferencias en FC, \% FCmáx, PSE y número de CJ. Específicamente, el JR3 fue la modalidad que generó mayores niveles de estas variables. Este efecto permite que los JR constituyan un contexto de trabajo adecuado para adaptar el ejercicio en función de los objetivos que se persigan.

La FC y el \% FCmáx mostraron tasas más elevadas en el JR3 que en los JR4 y JR5, aunque las diferencias fueron mayores entre el JR3 y el JR5. Estos resultados se situarían en la línea de otros trabajos realizados en fútbol (Abrantes y col., 2012; Owen y col., 2011), baloncesto (Castagna y col., 2011; Sampaio y col., 2009) o balonmano (Clemente y col., 2014), en el que los JR con un menor número de jugadores provocaba un incremento en la $\mathrm{FC}$ de los jugadores. En rugby, los resultados encontrados en el presente estudio coinciden parcialmente con lo encontrado por Foster y col. (2010), dado que hallaron resultados similares en un grupo de $15 \mathrm{y}$ 16 años, aunque no en el grupo de 12-13 años. A pesar de necesitar un mayor número de trabajos en rugby sobre este aspecto, los resultados hallados apoyan las conclusiones de aquellos estudios que establecen el incremento de la FC cuando los formatos de los JR tienen un menor número de jugadores (Hill-Haas et al., 2009; Katis and Kellis, 2009; Owen, Twist, y Ford, 2004; Rampinini y col., 2007), sugiriendo que se trata de un efecto bastante estable independientemente del deporte colectivo del que se trate.

La PSE y las CJ han descrito una tendencia similar aunque sensiblemente diferente a lo 
Muñoz-Chaves, B.M.; Reigal, R.E.; Hernández-Mendo, A., y Raimundi, M.J. (2015). Efectos del número de jugadores sobre la percepción subjetiva del esfuerzo, la frecuencia cardiaca y las conductas de juego en rugby. RICYDE. Revista internacional de ciencias del deporte, 42(11), 360-375.

http://dx.doi.org/10.5232/ricyde2015.04205

observado en FC, dado que no ha existido una relación lineal entre los tres juegos propuestos, contrario a lo mostrado en otros estudios como los de Owen y col. (2004) o Rampinini y col. (2007) en los que sí se había observando esta tendencia. No obstante, el JR3 es el que desarrolla tasas de PSE mayores que el JR4 y el JR5, lo cual se aproximaría a lo hallado en estudios efectuados en otros deportes colectivos en los que la PSE fue mayor en juegos con el menor número de jugadores (Abrantes y col., 2012; Morales y Arias-Estero, 2015). Sin embargo, datos encontrados en estudios como el de Sampaio y col. (2009) en jóvenes jugadores de baloncesto, en el que no encontraron diferencias significativas entre el JR3 y JR4, sugiere que es difícil establecer un comportamiento consistente en un amplio conjunto de casos. Por ello, aunque parece que modalidades con menor número de jugadores, como el JR3, produce niveles más elevados de PSE, sería aconsejable un análisis más exhaustivo y una mayor diferenciación entre tipos de deportes colectivos.

De igual modo, se esperaba que los JR con menor número de jugadores incrementara el número de acciones de juego (Duarte y col., 2009). Aunque el JR3 ha desarrollado más CJ que los JR4 y JR5, lo cual coincide con las apreciaciones de otros autores (Kelly y Drust, 2009; Klusemann y col., 2012; Owen y col., 2011), no se han encontrado diferencias significativas entre el JR4 y el JR5. Además, tal y como se ha obtenido en otros trabajos, existen diferencias en el comportamiento de las diversas CJ analizadas, lo que implica la necesidad de valorar de manera diferenciada cada una de ellas. Por lo tanto, estos hallazgos coinciden con los estudios de Owen y col. (2014) y Katis y Kellis (2009) en fútbol, en el que las modalidades con menor número de jugadores generaban más acciones en CJ como el pase corto y los lanzamientos, los cuales podrían relacionarse con el número de ensayos, siendo ambas acciones finalizaciones en la etapa de ataque. No obstante, no coincide plenamente con el estudio de Da Silva y col. (2011), pues aunque observaron en una muestra de jugadores adolescentes de fútbol que el formato 3 vs 3 (JR3) generaba un mayor número de lanzamientos que el 4 vs 4 (JR4) y el 5 vs 5 (JR5), no hubo diferencias en el pase.

Por tanto, aunque se aprecia que los JR con menor número de jugadores incrementan la FC, la PSE y las CJ, existen particularidades entre el JR4 y el JR5 que requieren mayor investigación en este deporte. Quizás una posible explicación de los resultados en PSE pudieran deberse al comportamiento de las acciones de juego. A pesar de no ser significativas, hubo diferencias a favor del JR5 en número de pases, placajes, rucks o continuidades, entre otras CJ. Esta mayor cantidad de acciones motrices que suceden en el JR5 coinciden con las encontradas por Villarejo y col. (2011), Villarejo y col. (2012) y Villarejo y col. (2015) para pases y placajes en partidos internacionales. Estos datos sugieren que el JR5 cubre las demandas técnicas y tácticas que se dan en un partido. Por otro lado, Estos procesos requieren un intenso y continuo esfuerzo cognitivo para atender las demandas técnico-tácticas de las tareas, así como elaborar la respuesta más adecuada, lo que podría influir en la fatiga percibida por el participante (Araújo, 2013; Tenenbaum, Basevitch, Gershgoren, y Filho, 2013; Vilar, Araújo, Davids, y Button, 2012). Aunque no puede confirmarse con los datos obtenidos, será una hipótesis a resolver en futuros estudios y podría justificar el diferente comportamiento presentado por la PSE y la FC en este trabajo.

Este estudio presenta una serie de limitaciones. En primer lugar, el tamaño de la muestra no permite generalizar los resultados encontrados. En futuros trabajos se debería ampliar el número de participantes para evaluar si se consolidan los resultados encontrados. Además, sería interesante analizar si los resultados encontrados en función de variables como el género, la posición del jugador, el nivel competitivo o la edad, para observar si son estables las diferencias encontradas. Por otro lado, sería interesante analizar JR con otras estructuras, diferente número 
Muñoz-Chaves, B.M.; Reigal, R.E.; Hernández-Mendo, A., y Raimundi, M.J. (2015). Efectos del número de jugadores sobre la percepción subjetiva del esfuerzo, la frecuencia cardiaca y las conductas de juego en rugby. RICYDE. Revista internacional de ciencias del deporte, 42(11), 360-375.

http://dx.doi.org/10.5232/ricyde2015.04205

de jugadores y otros tamaños de terrenos de juego. Esto ayudaría a evaluar con una mayor perspectiva la variabilidad observada en FC, \% FCmáx, PSE y CJ. Asimismo, una limitación importante de este trabajo deriva de la carencia de evidencias en este deporte de los efectos de los JR sobre las variables analizadas, lo que dificulta su discusión. En un futuro, posibles investigaciones similares ayudarán a consolidar o matizar los resultados hallados.

Sin embargo, a pesar de las limitaciones señaladas, este trabajo aporta datos interesantes sobre el efecto de los JR en rugby sobre la FC, la PSE y las CJ, deporte sobre el cual no existe un número elevado de evidencias. Aunque no existe un comportamiento generalizable y lineal entre los diferentes JR, se aprecia que el formato JR3 es el que genera mayores niveles en las variables analizadas, lo que sí es consistente con otras investigaciones efectuadas en otros deportes. Además, sugiere la relación entre las CJ desarrolladas y la PSE, lo que propone profundizar en este fenómeno en el contexto de los JR. Por último, este estudio aporta conocimientos que pueden ser útiles para los entrenadores en este deporte, ya que pueden utilizar esta información para adaptar el tipo de JR requerido para sus objetivos, mejorando el trabajo efectuado con sus jugadores.

\section{Referencias}

Abrantes, C.; Nunes, M.; Maças, V.; Leite, N., \& Sampaio, J. (2012). Effects of the number of players and game type constraints on heart rate, rating of perceived exertion and technical actions of small-sided soccer games. Journal of Strength and Conditioning Research, 26(4), 976-981.

http://dx.doi.org/10.1519/JSC.0b013e31822dd398

Almeida, CH.; Ferreira, AP., \& Volossovitch, A. (2012). Manipulating task constraints in small-sided soccer games: Performance analysis and practical implications. Open Sport Science Journal, 5, 174-180.

http://dx.doi.org/10.2174/1875399X01205010174

Araújo, D. (2013). The study of decision-making behavior in sport. RICYDE. Revista Internacional de Ciencias del Deporte, 9(31), 1-4.

Aroso, J.; Rebelo, N., \& Gomes-Pereira, J. (2004). Physiological impact of selected gamerelated exercises. Journal of Sports Sciences, 22(6), 522.

Ato, M.; López-García, J.J., y Benavente, A. (2013). Un sistema de clasificación de los diseños de investigación en psicología. Anales de Psicología, 29(3), 1038-1059. http://dx.doi.org/10.6018/analesps.29.3.178511

Blanco-Villaseñor, Á.; Castellano, J.; Hernández-Mendo, A.; Sánchez-López, C.R., y Usabiaga, O. (2014). Aplicación de la TG en el deporte para el estudio de la fiabilidad, validez y estimación de la muestra. Revista de Psicología del Deporte, 23(1), 131-137.

Bompa, T. (1993). Theory and methodology of training: the key to athletics performance. Iowa, Kendal/Hunt: Publishing Company.

Borg G.; Hassmen P., \& Lagerstrom M. (1987). Perceived exertion related to heart rate and blood lactate during arm and leg exercise. European Journal of Applied Physiology and Occupational Physiology, 56, 679-685.

http://dx.doi.org/10.1007/BF00424810

Casamichana, D.; Castellano, J., y Hernández-Mendo, A. (2014). La Teoría de la Generalizabilidad aplicada al estudio del perfil físico durante juegos reducidos con diferente orientación del espacio en fútbol. RICYDE. Revista Internacional de Ciencias del Deporte, 10(37), 194-205.

http://dx.doi.org/10.5232/ricyde2014.03702 
Muñoz-Chaves, B.M.; Reigal, R.E.; Hernández-Mendo, A., y Raimundi, M.J. (2015). Efectos del número de jugadores sobre la percepción subjetiva del esfuerzo, la frecuencia cardiaca y las conductas de juego en rugby. RICYDE. Revista internacional de ciencias del deporte, 42(11), 360-375.

http://dx.doi.org/10.5232/ricyde2015.04205

Casamichana, D.; Castellano, J.; González-Morán, A.; García-Cueto, H., y García-López, J. (2011). Demanda fisiológica en juegos reducidos de fútbol con diferente orientación del espacio. RICYDE. Revista Internacional de Ciencias del Deporte, 23(7), 141-154. http://dx.doi.org/10.5232/ricyde2011.02306

Casamichana, D., \& Castellano, J. (2010). Time-motion, heart rate, perceptual and motor behavior demands in small-sides soccer games: Effects of pitch size. Journal of Sports Sciences, 28(14), 1615-1623.

http://dx.doi.org/10.1080/02640414.2010.521168

Castagna, C.; Impellizzeri, F.; Chaouachi, A.; Abdelkrim, N., \& Manzi, V. (2011). Physiological responses to ball-drills in regional level male basketball players. Journal of Sports Sciences, 29(12), 1329-1336.

http://dx.doi.org/10.1080/02640414.2011.597418

Clemente, F.M.; Rocha, R.F.; Lourenço, F.M., \& Sousa, R. (2014). Acute effects of different formats of small-sided and conditioned handball games on heart rate responses in female students during PE classes. Sports, 2(2), 51-58. http://dx.doi.org/10.3390/sports2020051

Corvino, M.; Tessitore, A.; Minganti, C., \& Sibila, M. (2014). Effect of court dimensions on players' external and internal load during small-sided handball games. Journal of Sports Science \& Medicine, 13(2), 297.

Coutts, A.; Rampinini, E.; Marcora, S.M.; Castagna, C., \& Impellizzeri, FM. (2009). Heart rate and blood lactate correlates of perceived exertion during small-sided soccer games. Journal Science Medicine Sport, 12, 79-84.

http://dx.doi.org/10.1016/j.jsams.2007.08.005

Coutts, A.; Reaburn, P., \& Abt, G. (2003). Heart rate, blood lactate concentration and estimated energy expenditure in a semiprofessional rugby league team during a match: A case study. Journal Sports Science, 21, 97-103.

http://dx.doi.org/10.1080/0264041031000070831

Da Silva, C.D.; Impellizzeri, F.M.; Natali, A.J.; de Lima, J.R.; Bara-Filho, M.G.; SilamiGarçia, E., \& Marins, J. C. (2011). Exercise intensity and technical demands of smallsided games in young Brazilian soccer players: Effect of number of players, maturation, and reliability. The Journal of Strength \& Conditioning Research, 25(10), 2746-2751. http://dx.doi.org/10.1519/JSC.0b013e31820da061

Dellal, A.; Chamari, K.; Pintus, A.; Girard, O.; Cotte, T., \& Keller D. (2008). Heart rate responses during small-sided and short intermittent running training in elite soccer players: A comparative study. Journal of Strength and Conditioning Research, 22(5), 1449-1457.

http://dx.doi.org/10.1519/JSC.0b013e31817398c6

Dellal, A.; Chamari, K.; Pintus, A.; Girard, O.; Cotte, T., \& Keller, D. (2008). Heart rate responses during small-sided games and short intermittent running training. Journal of Strength and Conditioning Research, 22(5), 1449-1457.

http://dx.doi.org/10.1519/JSC.0b013e31817398c6

Dellal, A.; Drust, B., \& Lago-Penas, C. (2012). Variation of activity demands in small-sided soccer games. International Journal Sports Medicine, 33(5), 370-375. http://dx.doi.org/10.1055/s-0031-1295476

Dellal, A.; Owen, A.; Wong, D.P.; Krustrup, P.; van Excel, M., \& Mallo, J. (2009). Technical and physical demands of small vs. large sided games in relation to playing position in elite soccer. Human Movement Science Journal, 31, 957-969. http://dx.doi.org/10.1016/j.humov.2011.08.013 
Muñoz-Chaves, B.M.; Reigal, R.E.; Hernández-Mendo, A., y Raimundi, M.J. (2015). Efectos del número de jugadores sobre la percepción subjetiva del esfuerzo, la frecuencia cardiaca y las conductas de juego en rugby. RICYDE. Revista internacional de ciencias del deporte, 42(11), 360-375.

http://dx.doi.org/10.5232/ricyde2015.04205

Dellal, A.; Owen, D.P.; Wong, P.; Krustrup, M.; Exsel, V., \& Mallo, J. (2012). Technical and physical demands of small vs. large sided games in relation to playing position in elite soccer. Human Movement Science, 31(4), 957-969.

http://dx.doi.org/10.1016/j.humov.2011.08.013

Duarte, R.; Batalha, N.; Folgado, H., y Sampaio, J. (2009). Effects of exercise duration and number of players in heart rate responses and technical skills during futsal small-sided games. The Open Sports Sciences Journal, 2, 37-41. http://dx.doi.org/10.2174/1875399X00902010037

Estell, J.; Lord, P.; Barnsley, L.; Shenstone, B., \& Kannangara, S. (1996). The physiological demands of rugby league. In: Proceedings of the Australian Conference of Science and Medicine in Sport. Sports Medicine Australia, 2, 388-389.

Fanchini, M.; Azzalin, A.; Castagna, C.; Schena, F.; Mccall, A., \& Impellizzeri, F. (2011). Effect of bout duration on exercise intensity and technical performance of small-sided games in soccer. Journal of Strength and Conditioning Research, 25(2), 453-458. http://dx.doi.org/10.1519/JSC.0b013e3181c1f8a2

Feriche, B.; Chicharro, J. L.; Vaquero, A. F.; Perez, M., \& Lucia, A. (1998). The use of a fixed value of RPE during a ramp protocol. Comparison with the ventilatory threshold. The Journal of Sports Medicine and Physical Fitness, 38(1), 35-38.

Foster, C.D.; Twist, C.; Lamb, K.L., \& Nicholas, C. W. (2010). Heart rate responses to small-sided games among elite junior rugby league players. The Journal of Strength \& Conditioning Research, 24(4), 906-911. http://dx.doi.org/10.1519/JSC.0b013e3181aeb11a

Frencken, W.; Van Der Plaats, J.; Visscher, C., \& Lemmink, K. (2013). Size matters: Pitch dimensions constrain interactive team behaviour in soccer. Journal of Systems Science and Complexity, 26(1), 85-93.

http://dx.doi.org/10.1007/s11424-013-2284-1

Gabbett, T.J. (2006). Skill-based conditioning games as an alternative to traditional conditioning for rugby league players. The Journal of Strength \& Conditioning Research, 20(2), 306-315.

http://dx.doi.org/10.1519/00124278-200605000-00013

Gabbett, T.J.; Abernethy, B., \& Jenkins, D.G. (2012). Influence of field size on the physiological and skill demands of small-sided games in junior and senior rugby league players. The Journal of Strength \& Conditioning Research, 26(2), 487-491. http://dx.doi.org/10.1519/JSC.0b013e318225a371

Gabbett, T.; Jenkins, D.G., \& Abernethy, B. (2009). Game-based training for improving skill and physical fitness in team sport athletes. International Journal of Sports Science and Coaching, 4(2), 273-283.

http://dx.doi.org/10.1260/174795409788549553

Hill-Haas, S.; Coutts, A.; Roswell, G., \& Dawson, B. (2008). Variability of acute physiological responses and performance profiles of youth soccer players in small-sided games. Journal Science Medicine of Sport, 11, 487-490.

http://dx.doi.org/10.1016/j.jsams.2007.07.006

Hill-Haas, S.; Coutts, A. J.; Dawson, B. T., \& Rowsell, G. J. (2010). Time-motion characteristics and physiological responses of small-sided games in elite youth players: the influence of player number and rule changes. The Journal of Strength and Conditioning Research, 24(8), 2149-2156. http://dx.doi.org/10.1519/JSC.0b013e3181af5265 
Muñoz-Chaves, B.M.; Reigal, R.E.; Hernández-Mendo, A., y Raimundi, M.J. (2015). Efectos del número de jugadores sobre la percepción subjetiva del esfuerzo, la frecuencia cardiaca y las conductas de juego en rugby. RICYDE. Revista internacional de ciencias del deporte, 42(11), 360-375.

http://dx.doi.org/10.5232/ricyde2015.04205

Hill-Haas, S.; Dawson, B.; Coutts, A., \& Rowsell, G. (2009). Physiological responses and time-motion characteristics of various small-sided soccer games in youth players. Journal of Sports Sciences, 27(1), 1-8. http://dx.doi.org/10.1080/02640410802206857

Hill-Haas, S.; Dawson, B.; Impellizzeri, F.M., y Coutts, A. (2011). Physiology of small sided games training in football. A systematic review. Sports Medicine, 41(3), 199- 200. http://dx.doi.org/10.2165/11539740-000000000-00000

Hoff, J.; Wisloff, U.; Engen, L.C.; Kemi, O.J., y Helgerud, J. (2002). Soccer specific aerobic endurance training. British Journal Sports Medicine, 36, 218-221. http://dx.doi.org/10.1136/bjsm.36.3.218

Hoff, J., y Helgerud, J. (2004). Endurance and strength training for soccer players. Physiological considerations. Sports Medicine, 34(3), 165-80. http://dx.doi.org/10.2165/00007256-200434030-00003

Impellizzeri, F.M.; Marcora, S.M.; Castagna, C.; Reilly, T.; Sassi, A.; Iaia, F.M. y Rampinini, E. (2006). Physiological and performance effects of generic versus specific aerobic training in soccer players. International Journal Sports Medicine, 27, 483-492. http://dx.doi.org/10.1055/s-2005-865839

Jones, S., y Drust, B. (2007). Physiological and technical demands of $4 \vee 4$ and $8 \vee 8$ in elite youth soccer players. Kinesiology, 39(2), 150-156.

Katis, A., y Kellis, E. (2009). Effects of small-sided games on physical conditioning and performance in young soccer players. Journal of Sports Science and Medicine, 8(3), 374-380.

Kelly, D.M., y Drust, B. (2009). The effect of pitch dimensions on heart rate responses and technical demands of small-sided soccer games in elite players. Journal of Science and Medicine in Sport, 12(4), 475-479.

http://dx.doi.org/10.1016/j.jsams.2008.01.010

Klusemann, M.J.; Pyne, D.B.; Foster, C., y Drinkwater, E.J. (2012). Optimising technical skills and physical loading in small-sided basketball games. Journal of Sports Sciences, 30(14), 1463-1471.

http://dx.doi.org/10.1080/02640414.2012.712714

Köklü, Y.; Albayrak, M.; Keysan, H.; Alemdaroghe, U., y Dellal, A. (2013). Improvement of the physical conditioning of young soccer players by playing small sided games on different pitch size special reference to physiological responses. Kinesiology, 45(1), 4147.

Little, T., y Williams, G. (2006). Suitability of soccer training drills for endurance training. Journal of Strength and Conditioning Research, 20(2), 316-319.

Morales, M.T., y Arias-Estero, J.L. (2015). Diferencias entre el juego 7 vs. 7 y el 4 vs. 4 en el balonmano escolar en relación al rendimiento, percepción del esfuerzo y la intencionalidad de práctica. Retos, 27, 34-39.

Morgan W.P. (1994). Psychological components of effort senses. Medicine Science Sports Exercise, 26, 1071-1077.

http://dx.doi.org/10.1249/00005768-199409000-00001

Ortega, E., Villarejo, D., y Palao, J. M. (2009). Differences in game statistics between winning and losing rugby teams in the Six Nations Tournament. Journal of Sports Science and Medicine, 8, 523-527.

Owen, A.L.; Wong, D.P.; McKenna, M., y Dellal, A. (2011). Heart rate responses and technical comparison between small-vs. large-sided games in elite professional soccer. The Journal of Strength \& Conditioning Research, 25(8), 2104-2110.

http://dx.doi.org/10.1249/00005768-199409000-00001 
Muñoz-Chaves, B.M.; Reigal, R.E.; Hernández-Mendo, A., y Raimundi, M.J. (2015). Efectos del número de jugadores sobre la percepción subjetiva del esfuerzo, la frecuencia cardiaca y las conductas de juego en rugby. RICYDE. Revista internacional de ciencias del deporte, 42(11), 360-375.

http://dx.doi.org/10.5232/ricyde2015.04205

Owen, A.L.; Wong, D.P.; Paul, D., \& Dellal, A. (2014). Physical and technical comparisons between various-sided games within professional soccer. International Journal of Sports Medicine, 35(4), 286-92.

Owen, A.; Twist, C., \& Ford, P. (2004). Small-sided games: the physiological and technical effect of altering pitch size and player numbers. Insight, 7(2), 50-53.

Parlebas, P. (2001). Juegos, deportes y sociedad. Léxico de praxiología motriz. Barcelona: Paidotribo

Rampinini, E.; Impellizzeri, F.M.; Castagna, C.; Abt, G.; Chamari, K.; Sassi, A., \& Marcora, SM. (2007). Factors influencing physiological responses to small-sided soccer games. Journal of Sports Sciences, 25(6), 659-666. http://dx.doi.org/10.1080/02640410600811858

Sampaio, J.E.; Lago, C.; Gonçalves, B.; Maçãs, V.M., \& Leite, N. (2014). Effects of pacing, status and unbalance in time motion variables, heart rate and tactical behaviour when playing 5-a-side football small-sided games. Journal of Science and Medicine in Sport, $17(2), 229-233$.

http://dx.doi.org/10.1016/j.jsams.2013.04.005

Sampaio, J.; Abrantes, C., \& Leite, N. (2009). Power, heart rate and perceived exertion responses to $3 \times 3$ and $4 \times 4$ basketball small-sided games. Revista de Psicología del Deporte, 18(3), 463-467.

Sampaio, J.; Garcia, G.; Maças, V.; Ibanez, J.; Abrantes, C., \& Caixinha P. (2007). Heart rate and perceptual responses to $2 \times 2$ and $3 \times 3$ small-sided youth soccer games. Journal Sports Science Medicine, 6(suppl. 10), 121-122.

Seitz, L.B.; Rivière, M.; de Villarreal, E.S., \& Haff, G.G. (2014). The athletic performance of elite rugby league players is improved after an 8-week small-sided game training intervention. The Journal of Strength \& Conditioning Research, 28(4), 971-975.

Sproule, J.; Kunalan, C.; Mcneill, M., \& Wright H. (1993). Validity of 20-MST for predicting VO2 max of adult Singaporean athletes. British Journal Sports Medicine, 27(3). 202204.

http://dx.doi.org/10.1136/bjsm.27.3.202

Tenenbaum, G.; Basevitch, I.; Gershgoren, L., \& Filho, E. (2013). Emotions-decisionmaking in sport: Theoretical conceptualization and experimental evidence. International Journal of Sport and Exercise Psychology, 11(2), 151-168. http://dx.doi.org/10.1080/1612197X.2013.773687

Tessitore, A.; Meeusen, R.; Piacentini, M.; Demarie, S., \& Capranica, L. (2006). Physiological and technical aspects of "6-a-side" soccer drills. Journal of Sports Medicine and Physical Fitness, 46(1), 36-43.

Vickery, W.; Dascombe, B.; Duffield, R.; Kellett, A., \& Portus, M. (2013). Battlezone: An examination of the physiological responses, movement demands and reproducibility of small-sided cricket games. Journal of Sports Sciences, 31 (1), 77-86. http://dx.doi.org/10.1080/02640414.2012.720706

Vilar, L.; Araújo, D.; Davids, K., \& Button, C. (2012). The role of ecological dynamics in analysing performance in team sports. Sports Medicine, 42(1), 1-10. http://dx.doi.org/10.2165/11596520-000000000-00000

Villarejo, D., Palao, J. M., y Ortega, E. (2011). Diferencias en las estadísticas de juego entre equipos ganadores y perdedores en la Copa del Mundo de Rugby 2007. Kronos Rendimiento en el Deporte, 10(2), 41-47.

Villarejo, D., Palao, J. M., Ortega, E., Gomez-Ruano, M., \& Kraak, W. (2015). Match-related statistics discriminating between playing positions during the men's 2011 Rugby World Cup. International Journal of Performance Analysis in Sport, 15(1), 97-111. 
Muñoz-Chaves, B.M.; Reigal, R.E.; Hernández-Mendo, A., y Raimundi, M.J. (2015). Efectos del número de jugadores sobre la percepción subjetiva del esfuerzo, la frecuencia cardiaca y las conductas de juego en rugby. RICYDE. Revista internacional de ciencias del deporte, 42(11), 360-375.

http://dx.doi.org/10.5232/ricyde2015.04205

Wein, H. (1995). Fútbol a la medida del niño vol.1.Madrid: Gymnos.

Williams, K., y Owen, A. (2007). The impact of players numbers on the physiological responses to small sided games. Journal of Sports Science and Medicine, 6 (Suppl.10), 100-101. 